\title{
Stability control of fractional chaotic systems based on a simple Lyapunov function
}

\author{
Tianzeng Li ${ }^{\mathrm{a}, *}$, Yu Wang ${ }^{\mathrm{b}}$, Hongmei $\mathrm{Li}^{\mathrm{c}}$ \\ a School of Mathematics and Statistics, Artificial Intelligence Key Laboratory of Sichuan Province, Sichuan university of Science and \\ Engineering, Zigong 643000, China. \\ ${ }^{b}$ School of Mathematics and Statistics, Key Laboratory of Higher Education of Sichuan Province for Enterprise Informationalization and \\ Internet of Things, Sichuan university of Science and Engineering, Zigong 643000, China. \\ 'School of Economic and Management, Northwest University, Xi'an 710069, China.
}

Communicated by R. Saadati

\begin{abstract}
In this paper the stabilization of fractional-order chaotic systems and a new property of fractional derivatives are studied. Then we propose a new fractional-order extension of Lyapunov direct method and a control method based on a simple Lyapunov candidate function. The proposed control method can be applied to the stabilization of fractional-order chaotic and hyperchaotic systems. This control method is simple, universal, and theoretically rigorous. Numerical simulations are given for three fractional-order chaotic (or hyperchaotic) systems to verify the effectiveness and the universality of the proposed control method. (C)2017 All rights reserved.
\end{abstract}

Keywords: Lyapunov function, fractional-order, stabilization.

2010 MSC: 93D05, 93D15.

\section{Introduction}

Fractional-order dynamic systems, which are as a generalization of integer-order dynamic systems, provide better mathematical models for some actual physical and engineering systems [4, 30, 31]. We can find numerous applications in electrochemistry, viscoelasticity, porous media, control, and electromagnetic $[2,3,5,6,37]$. The fractional-order nonlinear dynamic systems have many dynamic behaviors which are similar to the integer-order systems, such as bifurcation, chaos, and attractor [13, 17, 24, 43]. The fractional-order chaotic systems are widely studied due to their potential applications in information, physics, chemistry, biology, and other fields [10, 14, 47]. Meanwhile, hyperchaotic systems are also considered with quickly increasing interest. It is usually defined as a chaotic system with more than one positive Lyapunov exponent, which implies that its dynamics are expended in several different directions simultaneously. Hence it has more complex dynamical behaviors than chaotic system. Typical examples

\footnotetext{
${ }^{*}$ Corresponding author

Email addresses: litianzeng27@163.com (Tianzeng Li), wangyu_813@163.com (Yu Wang), hmli@nwu.edu.cn (Hongmei Li)
} doi:10.22436/jnsa.010.09.28 
are four-dimensional hyperchaotic Chen's system [20], hyperchaotic Lorenz system [36], and hyperchaotic Rössler system [22, 32].

As we know, chaos control is very important but also very difficult in both chaotic systems and hyperchaotic systems. Some control methods are proposed to control fractional-order chaotic systems $[1,17,46]$, such as linear feedback controller [17], backstepping controller [1], sliding mode method [46], and the Lyapunov equation based method [19]. In all these cases, the stability of the whole controlled system have to be analyzed using the fractional-order techniques as well. For the fractional-order linear time invariant systems, the stability can be easily proved using the method proposed by Matignon [28]. The stability of a fractional-order nonlinear time varying system is proposed by Diethelm [9], but the result is valid only for scalar fractional-order systems. Hence, in order to prove the stability of fractionalorder nonlinear and time varying systems, some other methods must be applied. The important one of these methods is the fractional-order extension of Lyapunov direct method, which is proposed by Li et al. [18]. And in [39] the authors proposed Lyapunov direct method to prove the stability of fractionalorder nonlinear system with time delay. However, it is difficult to find a suitable Lyapunov candidate function. Some authors have presented Lyapunov functions to prove the stability of fractional-order systems $[15,16]$. And some Lyapunov functions have been proposed in papers related to fractional-order sliding mode control [7]. But we find that the Lyapunov functions which are proposed in these papers are not simple, and they are valid for fractional-order system with specific characteristics.

In this paper, we propose a new property of fractional-order derivatives which can help us to find a simple Lyapunov function. Then we propose a new fractional-order extension of Lyapunov direct method and a control method to control an arbitrary fractional-order chaotic system. Some sufficient conditions of controlling the fractional-order chaotic systems are proposed. The proposed control method is simple, universal and theoretically rigorous. Numerical simulation results of the control of the fractional-order unified system, the fractional-order Liu's system, and the hyperchaotic Chen system demonstrate the effectiveness and the universality of the proposed control method.

This paper is organized in the following manner. In Section 2 the preliminaries and some definitions are presented. Some control criteria of fractional-order chaotic systems are proposed in Section 3. In Section 4, numerical simulation of the systems shows the effectiveness and the universality of the control method. Finally, conclusions are in Section 5.

\section{Preliminaries and definitions}

\subsection{Fractional derivative and numerical solution of differential equation}

Fractional calculus plays an important role in modern science [31,38]. There are some definitions for fractional derivatives. In this paper, we give two commonly used definitions: Riemann-Liouville (RL), and Caputo and Grünwald-Letnikov definition [30, 34].

Definition 2.1. The fractional integral ${ }_{a} D_{t}^{-\alpha}$ of function $f(t)$ is defined as

$$
{ }_{a} D_{t}^{-\alpha} f(t)=\frac{1}{\Gamma(\alpha)} \int_{a}^{t}(t-\tau)^{\alpha-1} f(\tau) d \tau,
$$

where fractional order $\alpha>0$ and $\Gamma(z)=\int_{0}^{\infty} t^{z-1} e^{-t} d t$ is the gamma function.

Definition 2.2. The Caputo derivative with order $\alpha$ of function $f(t)$ is given as

$$
{ }_{a}^{C} D_{t}^{\alpha} f(t)={ }_{0} D_{t}^{-(n-\alpha)} \frac{d^{n}}{d t^{n}} f(t)=\frac{1}{\Gamma(n-\alpha)} \int_{a}^{t}(t-\tau)^{n-\alpha-1} f^{(n)}(\tau) d \tau,
$$

where $n-1<\alpha<n, n \in Z^{+}$. 
Definition 2.3. The Riemann-Liouville derivative with order $\alpha$ of function $f(t)$ is defined as

$$
{ }_{a}^{R L} D_{t}^{\alpha} f(t)=\frac{d^{n}}{d t^{n}}{ }_{a} D_{t}^{-(n-\alpha)} f(t)=\frac{1}{\Gamma(n-\alpha)} \frac{d^{n}}{d t^{n}} \int_{a}^{t}(t-\tau)^{n-\alpha-1} f(\tau) d \tau,
$$

where $n-1<\alpha<n, n \in Z^{+}$.

Definition 2.4. The Grünwald-Letnikov derivative with order $\alpha$ of function $f(t)$ is defined as

$$
\begin{aligned}
\underset{a}{G L} D_{t}^{\alpha} f(t) & =\lim _{\substack{h \rightarrow 0 \\
m h=t}} h^{-\alpha} \sum_{r=0}^{m}(-1)^{r}\left(\begin{array}{c}
\alpha \\
r
\end{array}\right) f(t-r h) \\
& =\sum_{k=0}^{n-1} \frac{f^{(k)}(0) t^{k-\alpha}}{\Gamma(k+1-\alpha)}+\frac{1}{\Gamma(n-\alpha)} \cdot \int_{a}^{t}(t-\tau)^{n-\alpha-1} f^{(n)}(\tau) d \tau,
\end{aligned}
$$

where $n-1<\alpha<n$.

It follows the definitions of fractional derivatives that the Grünwald-Letnikov fractional derivatives and Riemann-Liouville derivatives are equivalent. The Riemann-Liouville and Caputo definition are not equivalent, and their relation is expressed by

$$
{ }_{a}^{C} D_{t}^{\alpha} f(t)={ }_{a}^{R L} D_{t}^{\alpha} f(t)-\sum_{k=0}^{n-1} \frac{(t-a)^{k-\alpha} f^{(k)}(a)}{\Gamma(k-\alpha+1)} .
$$

Due to the relation (2.2), the Caputo derivative is equivalent to the Riemann-Liouville derivative if the initial conditions $f^{(k)}(a)=0, k=0,1, \cdots, n-1$ hold. Then we have another relation as follows.

Lemma 2.5. Suppose function $\mathrm{f}(\mathrm{t}) \in \mathrm{C}^{\mathfrak{n}}[\mathrm{a}, \mathrm{T}]$, then

$$
{ }_{a}^{C} D_{t}^{\alpha} f(t)={ }_{a}^{R L} D_{t}^{\alpha}\left(f(t)-\sum_{k=0}^{n-1} \frac{(t-a)^{k} f^{k}(a)}{k !}\right),
$$

where $n-1<\alpha \leqslant n$.

Proof. The relation (2.3) can be proved by the relation (2.2) and the definition of Caputo derivative. Let

$$
g(t)=f(t)-\sum_{k=0}^{n-1} \frac{(t-a)^{k} f^{(k)}(a)}{k !}
$$

where $g^{(k)}(a)=0, k=0,1, \cdots, n-1$.

It follows from relation (2.2) that ${ }_{a}^{C} D_{t}^{\alpha} g(t)={ }_{a}^{R L} D_{t}^{\alpha} g(t)$, i.e.,

$$
{ }_{a}^{C} D_{t}^{\alpha} g(t)={ }_{a}^{R L} D_{t}^{\alpha} g(t)={ }_{a}^{R L} D_{t}^{\alpha}\left(f(t)-\sum_{k=0}^{n-1} \frac{(t-a)^{k} f^{(k)}(a)}{k !}\right) .
$$

It follows from the definition of Caputo derivative that ${ }_{a}^{C} D_{t}^{\alpha}(t-a)^{k}=0$ with $0 \leqslant k<\alpha$. The left side of the equation (2.4) is equal to ${ }_{a}^{C} D_{t}^{\alpha} f(t)$, i.e.

$$
{ }_{a}^{C} D_{t}^{\alpha} g(t)={ }_{a}^{C} D_{t}^{\alpha} f(t) .
$$

Hence, the conclusion (2.3) is obtained. 
Given a discretization of $[a, T], a=t_{0}<t_{1}<\cdots<t_{N}=t$ with $t_{i+1}-t_{i}=h$, Riemann-Liouville and Grünwald-Letnikov fractional derivative can be approximated using the following formula,

$$
{ }_{a}^{R L} D_{t}^{\alpha} f(t)={ }_{a}^{G L} D_{t}^{\alpha} f(t)=\lim _{h \rightarrow 0} \frac{1}{h^{\alpha}} \sum_{k=0}^{N} c_{k}^{\alpha} f\left(t_{N-k}\right) \approx \frac{1}{h^{\alpha}} \sum_{k=0}^{N} c_{k}^{\alpha} f\left(t_{N-k}\right),
$$

and the Caputo fractional derivative can be approximated using the following relation

$$
{ }_{a}^{C} D_{t}^{\alpha} f(t) \approx \frac{1}{h^{\alpha}} \sum_{k=0}^{N} c_{j}^{\alpha}\left(f\left(t_{N-k}\right)-\sum_{j=0}^{n-1} \frac{(t-a)^{j} f^{(j)}(a)}{j !}\right),
$$

where $c_{j}^{\alpha}=(-1)^{j}\left(\begin{array}{l}\alpha \\ j\end{array}\right)$ are binomial coefficients.

This scheme is first introduced in [12] and known as GMMP scheme [44]. Based on this GMMP scheme (2.5), we give a numerical method of fractional differential equations. For explaining this method we consider the following equation:

$$
{ }_{a} D_{t}^{\alpha} x(t)=f(t, x(t)), \quad 0 \leqslant t \leqslant T, \quad x^{(k)}(a)=x_{0}^{(k)}, \quad k=0,1, \cdots, n-1,
$$

where ${ }_{a} D_{t}^{\alpha}$ denotes the fractional derivative of Riemann-Liouville (or Caputo) definition.

If ${ }_{a} D_{t}^{\alpha}$ is the Riemann-Liouville derivative, it follows from formula (2.5) that

$$
\sum_{k=0}^{N} c_{k}^{\alpha} x\left(t_{N-k}\right)=h^{\alpha} f\left(t_{N}, x\left(t_{N}\right)\right)
$$

i.e.,

$$
x\left(t_{N}\right)=h^{\alpha} f\left(t_{N}, x\left(t_{N}\right)\right)-\sum_{k=1}^{N} c_{k}^{\alpha} x\left(t_{N-k}\right) .
$$

If ${ }_{a} D_{t}^{\alpha}$ is the Caputo derivative, it follows formula (2.6) that

$$
\sum_{k=0}^{N} c_{j}^{\alpha}\left(x\left(t_{N-k}\right)-\sum_{j=0}^{n-1} \frac{(t-a)^{j} x^{(j)}(a)}{j !}\right)=h^{\alpha} f\left(t_{N}, x\left(t_{N}\right)\right),
$$

i.e.,

$$
x\left(t_{N}\right)=h^{\alpha} f\left(t_{N}, x\left(t_{N}\right)\right)+\sum_{j=0}^{n-1} \frac{(t-a)^{j} x^{(j)}(a)}{j !}-\sum_{k=1}^{N} c_{j}^{\alpha}\left(x\left(t_{N-k}\right)-\sum_{j=0}^{n-1} \frac{(t-a)^{j} x^{(j)}(a)}{j !}\right) .
$$

Especially, let $0<\alpha \leqslant 1$, the formula (2.7) can be simplified as follows:

$$
x\left(t_{N}\right)=h^{\alpha} f\left(t_{N}, x\left(t_{N}\right)\right)+x(a)-\sum_{k=1}^{N} c_{j}^{\alpha}\left(x\left(t_{N-k}\right)-x(a)\right) .
$$

Based on the Grünwald-Letnikov formula, an implicit difference scheme (2.8) has been presented. In the formula (2.8), the $x\left(t_{N}\right)$ is an unknown variable, which is in both sides of the nonlinear equation, then Newton-Raphson method can be used to solve the value of $x\left(t_{N}\right)$ by equation (2.8). In this paper, the GMMP scheme and Newton-Raphson method are used to obtain the numerical solution of the fractional systems.

\subsection{Some properties of the fractional derivative}

In this paper we mainly consider the fractional order $0<\alpha<1$. There are some general properties of the fractional-order derivative ${ }_{a} D_{t}^{\alpha}$ (Riemann-Liouville and Caputo definition), which are described as follows [30, 31]. 
Property 2.6 (Linearity [31]). Caputo fractional derivative is a linear operation, i.e.,

$$
{ }_{a} D_{t}^{\alpha}(\lambda f(t)+\mu g(t))=\lambda_{a} D_{t}^{\alpha} f(t)+\mu_{a} D_{t}^{\alpha} g(t),
$$

where $\lambda, \mu$ are real constants.

Property 2.7 (Leibniz rule for fractional differentiation $[30,31])$. If $f(\tau)$ and $\varphi(\tau)$ along with all its derivatives are continuous in $[a, t]$, the fractional derivative of the product $\varphi(t) f(t)$ is given by

$$
{ }_{a} D_{t}^{\alpha}(\varphi(t) f(t))=\sum_{k=0}^{\infty}\left(\begin{array}{l}
\alpha \\
k
\end{array}\right) \varphi^{(k)}(t)_{a} D_{t}^{\alpha-k} f(t) .
$$

In the following, two new properties of fractional-order derivatives are proposed, which can help us to find a simple Lyapunov function.

Property 2.8 (Caputo definition ${ }_{a}^{C} D_{t}^{\alpha}$ ). Let $x(t)=\left(x_{1}(t), \cdots, x_{n}(t)\right)^{\top} \in \mathbb{R}^{n}$ have a continuous derivative in $[a, t]$, for any positive definite matrix $P$, then

$$
{ }_{a}^{C} D_{t}^{\alpha}\left(\frac{1}{2} x^{\top}(t) P x(t)\right) \leqslant x^{\top}(t) P_{a}^{C} D_{t}^{\alpha} x(t), \quad \forall \alpha \in(0,1),
$$

where the ${ }_{a}^{C} D_{t}^{\alpha}$ denotes the Caputo fractional derivative.

Proof. Firstly, let

$$
f(t)={ }_{a}^{C} D_{t}^{\alpha}\left(\frac{1}{2} x^{\top}(t) P x(t)\right)-x^{\top}(t) P_{a}^{C} D_{t}^{\alpha} x(t),
$$

then proving that expression (2.9) is true is equivalent to prove that

$$
f(t)=\frac{1}{2}{ }_{a}^{C} D_{t}^{\alpha}\left(x^{\top}(t) P x(t)\right)-x^{\top}(t) P_{a}^{C} D_{t}^{\alpha} x(t) \leqslant 0 .
$$

Due to the Caputo definition (2.1), the function (2.10) can be written as

$$
\begin{aligned}
& f(t)=\frac{1}{\Gamma(1-\alpha)} \int_{a}^{t} \frac{\chi^{\top}(\tau) P \dot{x}(\tau)}{(t-\tau)^{\alpha}} d \tau-\frac{1}{\Gamma(1-\alpha)} x^{\top}(t) P \int_{a}^{t} \frac{\dot{x}(\tau)}{(t-\tau)^{\alpha}} d \tau \\
& =\frac{1}{\Gamma(1-\alpha)} \int_{a}^{t} \frac{x^{\top}(\tau) P \dot{x}(\tau)-x^{\top}(t) P \dot{x}(\tau)}{(t-\tau)^{\alpha}} d \tau \\
& =\frac{1}{\Gamma(1-\alpha)} \int_{a}^{t} \frac{\left(x^{\top}(\tau)-x^{\top}(t)\right) P \dot{x}(\tau)}{(t-\tau)^{\alpha}} d \tau \\
& \underline{\underline{y(\tau)=x(\tau)-x(t)}} \frac{1}{\Gamma(1-\alpha)} \int_{a}^{t} \frac{y^{\top}(\tau) P \dot{y}(\tau)}{(t-\tau)^{\alpha}} d \tau \\
& \underline{\underline{\mathbf{y}^{\top}(\tau) \mathrm{P} \dot{y}(\tau) \mathrm{d} \tau=\frac{1}{2} \mathrm{~d}\left(\boldsymbol{y}^{\top}(\tau) \mathrm{P} \boldsymbol{y}(\tau)\right)}} \frac{1}{2 \Gamma(1-\alpha)} \int_{a}^{t}(t-\tau)^{-\alpha} \mathrm{d}\left(\mathbf{y}^{\top}(\tau) \mathrm{P} \boldsymbol{y}(\tau)\right) .
\end{aligned}
$$

Let us integrate by parts of formula (2.12), the function $f(t)$ can be rewritten as

$$
\begin{aligned}
f(t) & =\left.\frac{1}{2 \Gamma(1-\alpha)} \frac{y^{\top}(\tau) P y(\tau)}{(t-\tau)^{\alpha}}\right|_{a} ^{t}-\frac{\alpha}{2 \Gamma(1-\alpha)} \int_{a}^{t} \frac{y^{\top}(\tau) P y(\tau)}{(t-\tau)^{\alpha+1}} d \tau \\
& =\left.\frac{y^{\top}(\tau) P y(\tau)}{2 \Gamma(1-\alpha)(t-\tau)^{\alpha}}\right|_{\tau=t}-\frac{y^{\top}(a) P y(a)}{2 \Gamma(1-\alpha)(t-a)^{\alpha}}-\frac{\alpha}{2 \Gamma(1-\alpha)} \int_{a}^{t} \frac{y^{\top}(\tau) P y(\tau)}{(t-\tau)^{\alpha+1}} d \tau .
\end{aligned}
$$

Let check the first term of formula (2.13), which has an indetermination at $\tau=t$, then we can analyze the corresponding limitation by the L'Hopital rule

$$
\lim _{\tau \rightarrow t} \frac{y^{\top}(\tau) P y(\tau)}{(t-\tau)^{\alpha}}=\lim _{\tau \rightarrow t} \frac{2 y^{\top}(\tau) P \dot{y}(\tau)}{-\alpha(t-\tau)^{\alpha-1}}=\lim _{\tau \rightarrow t} \frac{2 y^{\top}(\tau) P \dot{y}(\tau)(t-\tau)^{1-\alpha}}{-\alpha}=0 .
$$


And the matrix $P$ is positive definite, then

$$
\frac{\boldsymbol{y}^{\top}(a) P \boldsymbol{y}(a)}{2 \Gamma(1-\alpha)(t-a)^{\alpha}} \geqslant 0
$$

and

$$
\frac{\alpha}{2 \Gamma(1-\alpha)} \int_{a}^{t} \frac{y^{\top}(\tau) P y(\tau)}{(t-\tau)^{\alpha+1}} d \tau \geqslant 0
$$

Hence, we obtain that $f(t) \leqslant 0$, i.e., the conclusion (2.9) is clearly true.

Property 2.9 (Riemann-Liouville definition $\left.{ }_{a}^{R} D_{t}^{\alpha}\right)$. Let $x(t)=\left(x_{1}(t), \cdots, x_{n}(t)\right)^{\top} \in \mathbb{R}^{n}$ have a continuous derivative in $[a, t]$, for any positive definite matrix $P$, then

$$
{ }_{\mathrm{a}}^{\mathrm{R}} \mathrm{D}_{\mathrm{t}}^{\alpha}\left(\frac{1}{2} x^{\top}(\mathrm{t}) \mathrm{P} x(\mathrm{t})\right) \leqslant \boldsymbol{x}^{\top}(\mathrm{t}) \mathrm{P}_{\mathrm{a}}^{\mathrm{R}} \mathrm{D}_{\mathrm{t}}^{\alpha} \boldsymbol{x}(\mathrm{t}), \quad \forall \alpha \in(0,1),
$$

where the ${ }_{a}^{R} D_{t}^{\alpha}$ denotes the Riemann-Liouville fractional derivative.

Proof. Firstly, let

$$
f(t)={ }_{a}^{R} D_{t}^{\alpha}\left(\frac{1}{2} x^{\top}(t) P x(t)\right)-x^{\top}(t) P_{a}^{R} D_{t}^{\alpha} x(t),
$$

then proving that expression (2.10) is true is equivalent to prove that

$$
f(t)=\frac{1}{2}{ }_{a}^{R} D_{t}^{\alpha}\left(x^{\top}(t) P x(t)\right)-x^{\top}(t) P_{a}^{R} D_{t}^{\alpha} x(t) \leqslant 0 .
$$

Due to the Riemann-Liouville definition (2.3), the function (2.11) can be written as

$$
\begin{aligned}
f(t) & =\frac{1}{2 \Gamma(1-\alpha)} \frac{d}{d t} \int_{a}^{t} \frac{x^{\top}(\tau) P x(\tau)}{(t-\tau)^{\alpha}} d \tau-\frac{1}{\Gamma(1-\alpha)} x^{\top}(t) P \frac{d}{d t} \int_{a}^{t} \frac{x(\tau)}{(t-\tau)^{\alpha}} d \tau \\
& =\frac{1}{\Gamma(1-\alpha)}\left\{\frac{1}{2} \frac{d}{d t} \int_{a}^{t} \frac{x^{\top}(\tau) P x(\tau)}{(t-\tau)^{\alpha}} d \tau-x^{\top}(t) \frac{d}{d t} \int_{a}^{t} \frac{P x(\tau)}{(t-\tau)^{\alpha}} d \tau\right\} .
\end{aligned}
$$

Let

$$
g(t)=\frac{1}{2} \frac{d}{d t} \int_{a}^{t} \frac{x^{\top}(\tau) P x(\tau)}{(t-\tau)^{\alpha}} d \tau-x^{\top}(t) \frac{d}{d t} \int_{a}^{t} \frac{P x(\tau)}{(t-\tau)^{\alpha}} d \tau
$$

then

$$
\begin{aligned}
& g(t) \stackrel{y=t-\tau}{=} \frac{1}{2} \frac{d}{d t} \int_{0}^{t-a} \frac{x^{\top}(t-y) P x(t-y)}{y^{\alpha}} d y-x^{\top}(t) \frac{d}{d t} \int_{0}^{t-a} \frac{P x(t-y)}{y^{\alpha}} d y \\
& =\frac{1}{2} \frac{x^{\top}(a) P x(a)}{(t-a)^{\alpha}}+\int_{0}^{t-a} \frac{x^{\top}(t-y) P \dot{x}(t-y)}{y^{\alpha}} d y-x^{\top}(t)\left\{\frac{P x(a)}{(t-a)^{\alpha}}+\int_{0}^{t-a} \frac{P \dot{x}(t-u)}{y^{\alpha}} d y\right\} \\
& =\frac{1}{2} \frac{x^{\top}(a) P x(a)}{(t-a)^{\alpha}}-\frac{x^{\top}(t) P x(a)}{(t-a)^{\alpha}}+\int_{0}^{t-a} \frac{\left(x^{\top}(t-y)-x^{\top}(t)\right) P \dot{x}(t-y)}{y^{\alpha}} d y \\
& \underline{\underline{\tau=t-y}} \frac{1}{2} \frac{x^{\top}(a) P x(a)}{(t-a)^{\alpha}}-\frac{x^{\top}(t) P x(a)}{(t-a)^{\alpha}}+\int_{a}^{t} \frac{\left(x^{\top}(\tau)-x^{\top}(t)\right) P \dot{x}(\tau)}{(t-\tau)^{\alpha}} \\
& \underline{\underline{y(\tau)=x(\tau)-x(t)}} \frac{1}{2} \frac{x^{\top}(a) P x(a)}{(t-a)^{\alpha}}-\frac{x^{\top}(t) P x(a)}{(t-a)^{\alpha}}+\int_{a}^{t}(t-\tau)^{-\alpha} d\left(\frac{1}{2} y^{\top}(\tau) P y(\tau)\right) .
\end{aligned}
$$

Let us integrate by parts of formula (2.15), the function $g(t)$ can be rewritten as

$$
\begin{aligned}
g(t) & =\frac{1}{2} \frac{x^{\top}(a) P x(a)}{(t-a)^{\alpha}}-\frac{x^{\top}(t) P x(a)}{(t-a)^{\alpha}}+\left.\frac{1}{2} \frac{y^{\top}(\tau) P y(\tau)}{(t-\tau)^{\alpha}}\right|_{a} ^{t}-\frac{\alpha}{2} \int_{a}^{t} \frac{y^{\top}(\tau) P y(\tau)}{(t-\tau)^{\alpha+1}} d \tau \\
& =\frac{1}{2} \lim _{\tau \rightarrow t} \frac{y^{\top}(\tau) P y(\tau)}{(t-\tau)^{\alpha}}-\frac{x^{\top}(t) P x(t)}{2(t-a)^{\alpha}}-\frac{\alpha}{2} \int_{a}^{t} \frac{y^{\top}(\tau) P y(\tau)}{(t-\tau)^{\alpha+1}} d \tau .
\end{aligned}
$$


Let check the first term of formula (2.16), which has an indetermination at $\tau=t$, then we can analyze the corresponding limitation by the L'Hopital rule,

$$
\lim _{\tau \rightarrow t} \frac{y^{\top}(\tau) P y(\tau)}{(t-\tau)^{\alpha}}=\lim _{\tau \rightarrow t} \frac{2 y^{\top}(\tau) P \dot{y}(\tau)}{-\alpha(t-\tau)^{\alpha-1}}=\lim _{\tau \rightarrow t} \frac{2 y^{\top}(\tau) P \dot{y}(\tau)(t-\tau)^{1-\alpha}}{-\alpha}=0
$$

And the matrix $P$ is positive definite, then

$$
\frac{x^{\top}(a) P x(a)}{2(t-a)^{\alpha}} \geqslant 0
$$

and

$$
\frac{\alpha}{2} \int_{a}^{t} \frac{y^{\top}(\tau) P y(\tau)}{(t-\tau)^{\alpha+1}} d \tau \geqslant 0
$$

Hence, we obtain that $g(t) \leqslant 0$, i.e. $f(t) \leqslant 0$, then the conclusion $(2.14)$ is clearly true.

Remark 2.10. If the positive definite matrix is an identity matrix, i.e., $\mathrm{P}=\mathrm{I}$, Properties 2.8 and 2.9 can be written as

$$
{ }_{a} D_{t}^{\alpha}\left(\frac{1}{2} x^{\top}(t) x(t)\right) \leqslant x^{\top}(t)_{a} D_{t}^{\alpha} x(t), \quad \forall \alpha \in(0,1) .
$$

\subsection{Stability of fractional-order system}

The fractional-order nonlinear system which is given as

$$
{ }_{0} D_{t}^{\alpha} x(t)=f(t, x(t)),
$$

where $f=\left(f_{1}, f_{2}, \ldots, f_{n}\right)^{\top}$ is a vector function and $f_{i}(i=1,2, \ldots, n)$ are continuous differential nonlinear functions, $\alpha$ is the fractional order of derivative, $x(t)=\left(x_{1}(t), x_{2}(t), \cdots, x_{n}(t)\right)^{\top}$ is the system state variable, and ${ }_{0} D_{t}^{\alpha}$ denotes the Caputo fractional-order derivative operator. The equilibrium points of this system are calculated by solving $\mathbf{f}\left(\boldsymbol{x}^{*}\right)=0$. For this fractional-order nonlinear system, the fractional-order extension of Lyapunov direct method has been proposed, which is given as follows [18].

Theorem 2.11. Let $\mathrm{x}=0$ be an equilibrium point for the nonautonomous fractional-order system (2.17). Assume that there exists a Lyapunov function $\mathrm{V}(\mathrm{t}, \boldsymbol{x}(\mathrm{t}))$ and class-K functions $\alpha_{i}(i=1,2,3)$ satisfying

$$
\alpha_{1}(\|x(t)\|) \leqslant V(t, x(t)) \leqslant \alpha_{2}(\|x(t)\|), \quad{ }_{0} D_{t}^{\beta} V(t, x(t)) \leqslant-\alpha_{3}(\|x(t)\|),
$$

where $\beta \in(0,1)$. Then the equilibrium point of system (2.17) is asymptotically stable.

\section{Stability control of fractional chaotic system}

In this section, based on the fractional-order extension of the Lyapunov direct method and the new property of fractional derivatives, we find a suitable Lyapunov function and propose the control criterion of the fractional chaotic system.

Theorem 3.1. For the fractional-order system

$$
{ }_{0} D_{t}^{\alpha} x(t)=f(x(t)),
$$

where $\alpha \in(0,1)$, without loss of generality, let $\boldsymbol{x}^{*}=0$ is the equilibrium point and $\boldsymbol{x}(\mathrm{t})$ is the state variable. If there exists a positive definite matrix $\mathbf{P}$, which satisfies

$$
x^{\top}(t) \operatorname{Pf}(x(t)) \leqslant 0,
$$

then the origin of the system (3.1) is asymptotically stable. 
Proof. Since $\mathbf{P}$ is a positive definite matrix, we introduce a Lyapunov function

$$
\mathrm{V}(\boldsymbol{x}(\mathrm{t}))=\frac{1}{2} \boldsymbol{x}^{\top}(\mathrm{t}) \mathbf{P} \boldsymbol{x}(\mathrm{t})
$$

It follows from Properties 2.8 and 2.9 that

$$
{ }_{0} D_{t}^{\alpha} V(x(t)) \leqslant x^{\top}(t) P_{0} D_{t}^{\alpha} x(t)=x^{\top}(t) \operatorname{Pf}(x(t)) .
$$

And since $x^{\top}(t) \operatorname{Pf}(\boldsymbol{x}(t)) \leqslant 0$, we have the fractional-order derivative (3.2) of the Lyapunov function is negative definite. Using the relation between positive definite functions and class- $K$ functions in [33], it follows from Theorem 2.11 that the origin of the system (3.1) is asymptotically stable.

In the following, we would give the control criterion of the fractional chaotic system. Firstly we rewrite the fractional-order chaotic system (3.1) as follows

$$
{ }_{0} D_{t}^{\alpha} x(t)=f(x(t))=A x(t)+g(x(t)) x(t),
$$

where $A x(t)$ is the linear part of system (3.1), and $g(x(t)) x(t)$ is the nonlinear part of system (3.1). This way of writing is very general and almost all fractional-order chaotic systems can be written as the form (3.3).

For controlling the fractional-order chaotic system to its equilibrium points, we add linear feedback control input to system (3.3). As we know, the linear controller has many advantages: (1) it is very simple; (2) it is easily realized experimentally; (3) it is more suitable for engineering applications than other controllers.

Hence the controlled fractional-order chaotic system (3.3) with linear feedback control input is given as

$$
{ }_{0} D_{t}^{\alpha} x(t)=A x(t)+g(x(t)) x(t)-K x(t),
$$

where $\mathbf{K} x(t)$ is the linear feedback control input, and the feedback gain matrix $K \in \mathbb{R}^{n \times n}$ needs to be determined.

Therefore, our aim is to design a suitable feedback gain matrix $\mathrm{K}$ such that the controlled system (3.4) is asymptotically stable.

Theorem 3.2. The controlled fractional-order chaotic system (3.4) is asymptotically stable at the origin, if the feedback gain matrix $\mathbf{K}$ makes the symmetric matrix

$$
\mathbf{S}=\frac{(\mathbf{P A}+\mathbf{P g}(x)-\mathbf{P K})^{\top}+(\mathbf{P A}+\mathbf{P g}(x)-\mathbf{P K})}{2}
$$

negative definite for all $\boldsymbol{x}(\mathbf{t})$, where $\mathbf{P}$ is a positive definite.

Proof. For the controlled system (3.4), we introduce the Lyapunov function

$$
V(x)=\frac{1}{2} x^{\top}(t) P x(t)
$$

where $\mathrm{P}$ is a positive definite matrix. It follows from Property 2.8 that

$$
\begin{aligned}
{ }_{0} D_{t}^{\alpha} V(x) & =\frac{1}{2}{ }_{0} D_{t}^{\alpha}\left(x^{\top}(t) P x(t)\right) \\
& \leqslant x^{\top}(t) P_{0} D_{t}^{\alpha} x(t) \\
& =x^{\top}(t) P(A x(t)+g(x(t)) x(t)-K x(t)) \\
& =x^{\top}(t)(P A+P g(x(t))-P K) x(t) \\
& =x^{\top}(t) S x(t),
\end{aligned}
$$


where

$$
\mathbf{S}=\frac{(\mathbf{P A}+\mathbf{P g}(x)-\mathbf{P K})^{\top}+(\mathbf{P A}+\mathbf{P g}(x)-\mathbf{P K})}{2}
$$

is a symmetric matrix. If $S$ is negative definite for all $x(t)$, we have

$$
{ }_{0} \mathrm{D}_{\mathrm{t}}^{\alpha} \mathrm{V}(\boldsymbol{x}) \leqslant \boldsymbol{x}^{\top}(\mathrm{t}) \boldsymbol{S} \boldsymbol{x}(\mathrm{t})<0 .
$$

It follows from Theorem 3.1 that the controller can make the chaotic system asymptotically stable at the origin.

In this paper, we mainly consider the control of fractional-order chaotic system. As we know, for the fractional-order chaotic system (3.3), the state variable $x(t)$ is bounded. Then we obtain a conclusion: there is a constant matrix $\mathbf{C}$, satisfying

$$
x^{\top}(t) g(x(t)) x(t) \leqslant x^{\top}(t) C x(t) .
$$

Hence, we can give some corollaries which are simpler than Theorem 3.2.

Corollary 3.3. The controlled fractional-order chaotic system (3.4) is asymptotically stable at the origin, if the feedback gain matrix $\mathbf{K}$ makes the matrix

$$
\mathbf{S}=\frac{(\mathbf{P A}+\mathbf{P C}-\mathbf{P K})^{\mathrm{T}}+(\mathbf{P A}+\mathbf{P C}-\mathbf{P K})}{2}
$$

negative definite, where $\mathbf{P}$ is a positive definite and $\mathbf{C}$ is defined as (3.5).

This corollary can be easily proved by Theorem 3.2 and (3.5).

If we choose the positive definite matrix $\mathbf{P}=\mathbf{I}$, the constant matrix $\mathbf{C}=\mathbf{c I}$, and the feedback gain matrix $\mathbf{K}=\mathbf{k} \mathbf{I}$, where $\mathbf{I}$ is the identity matrix, the following corollary can be obtained.

Corollary 3.4. The controlled fractional-order chaotic system (3.4) is asymptotically stable at the origin, if the feedback gain matrix $\mathbf{K}=\mathrm{k} \mathbf{I}$ makes the matrix

$$
\mathbf{S}=\frac{\mathbf{A}^{\top}+\mathbf{A}}{2}+(\mathrm{c}-\mathrm{k}) \mathbf{I}
$$

negative definite. Particularly, let $\lambda_{\text {max }}$ be the maximal eigenvalue of the matrix $\frac{\mathbf{A}^{\top}+\mathbf{A}}{2}$, if $\mathbf{K}=\mathrm{k} \mathbf{I}$ satisfies

$$
\lambda_{\max }+\mathrm{c}-\mathrm{k}<0,
$$

the controlled system (3.4) is asymptotically stable at the origin.

Remark 3.5. In these corollaries, these control criteria are sufficient conditions for the stability of the fractional chaotic system. In the application, we only choose the feedback gain matrix $\mathbf{K}=k \mathbf{I}$, which satisfies $k>\lambda_{\max }+c$, then the controller can make the system (3.4) asymptotically stable at the origin. This method is simple and universal.

Remark 3.6. In Corollary 3.4, we suppose that the constant matrix is $\mathbf{C}=\mathbf{c I}$ and the feedback gain matrix is $\mathbf{K}=\mathbf{k I}$, then the conclusion is obtained. If we suppose that $\mathbf{C}$ and $\mathbf{K}$ are diagonal matrices, i.e., $\mathbf{C}=\operatorname{diag}\left(c_{1}, c_{2}, \cdots, c_{n}\right)$ and $\mathbf{K}=\operatorname{diag}\left(k_{1}, k_{2}, \cdots, k_{n}\right)$, according to Theorem 3.2 and Corollary 3.4, we must find suitable $k_{i}$ which satisfies the condition. In many cases, we would find some $k_{i}$ and $c_{i}$ are equal to zero, which make the linear controller simpler.

\section{Simulation and analysis}

In this section, two 3D fractional-order chaotic systems and one 4D fractional-order hyperchaotic system are used as examples to show the validity and effectiveness of the control criterion. For the fractional-order chaotic system as form (3.4), by adding a linear controller and letting the feedback gain matrix satisfy the conditions of Corollary 3.4, the chaotic system can be stabilized to the equilibrium point. We use the GMMP scheme and Newton-Raphson method to solve the numerical solution of the fractional systems. 


\subsection{Stabilization of the fractional-order unified system}

The unified system [26], which is introduced by Lü et al. in 2002, can unify the Lorenz, the Chen and the Lü system. The expressions for the fractional-order unified system is given by [21, 41]

$$
\begin{aligned}
& { }_{0} D_{t}^{\alpha} x_{1}=(25 a+10)\left(x_{2}-x_{1}\right), \\
& { }_{0} D_{t}^{\alpha} x_{2}=(28-35 a) x_{1}+(29 a-1) x_{2}-x_{1} x_{3}, \\
& { }_{0} D_{t}^{\alpha} x_{3}=x_{1} x_{2}-(a+8) / 3 x_{3},
\end{aligned}
$$

where $a \in R$ and $\alpha$ is the fractional order of derivatives. When $a \in[0,0.8)$, system (4.1) is the fractionalorder generalized Lorenz system; when $a=0.8$, it becomes the fractional-order Lü system; when $a \in$ $(0.8,1]$, it is the fractional-order generalized Chen system. If we select $a=1$, there are three equilibrium points $(0,0,0),(3 \sqrt{7}, 3 \sqrt{7}, 21)$, and $(-3 \sqrt{7},-3 \sqrt{7}, 21)$ in the system. With the fractional order $\alpha=0.9$ and initial value $(2,2,1)$, the unified system (3.3) can generate chaos which is shown in Fig. 1.
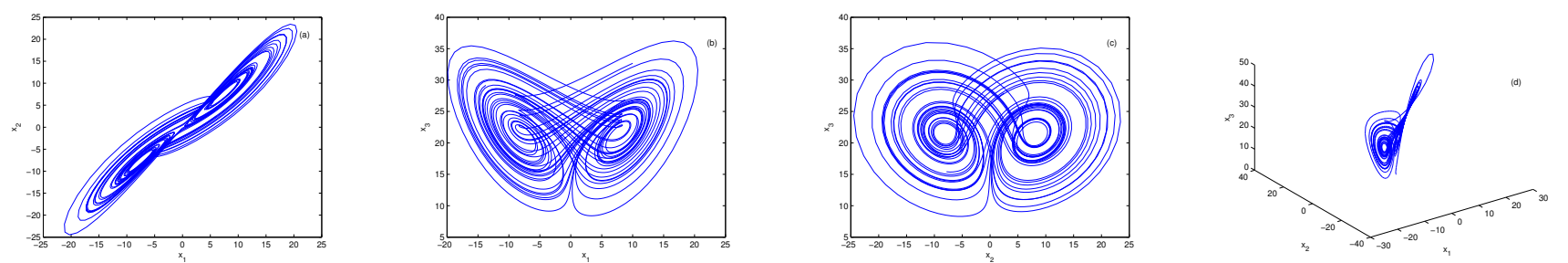

Figure 1: Chaotic attractors in the fractional-order unified system with $\alpha=0.9$. The panels (a), (b), (c), and (d) show the $x_{2}-x_{1}$, $x_{3}-x_{1}, x_{3}-x_{2}$, and $3 D$ views, respectively.

The controlled fractional-order chaotic system (4.1) can be rewritten as form (3.4),

$$
{ }_{0} D_{t}^{\alpha} x(t)=A x(t)+g(x(t)) x(t)-K x(t),
$$

where

$$
\mathbf{A}=\left(\begin{array}{ccc}
-35 & 35 & 0 \\
7 & 28 & 0 \\
0 & 0 & -3
\end{array}\right), \quad \mathbf{g}(\boldsymbol{x}(\mathbf{t}))=\left(\begin{array}{ccc}
0 & 0 & 0 \\
0 & 0 & -x_{1} \\
0 & x_{1} & 0
\end{array}\right)
$$

It follows by $\boldsymbol{x}^{\top}(\mathrm{t}) \mathbf{g}(\boldsymbol{x}(\mathrm{t})) \boldsymbol{x}(\mathrm{t})=0$ that $\mathbf{C}=0$. According to Corollary 3.4, if the matrix $\mathbf{S}=\frac{\mathbf{A}^{\top}+\mathbf{A}}{2}-\mathrm{kI}$ is negative positive, the system (4.2) is asymptotically stable. And we can easily obtain that the maximal eigenvalue of matrix $\frac{\mathbf{A}^{\top}+\mathbf{A}}{2}$ is $\lambda_{\max } \approx 31$. So if the the feedback gain matrix $\mathbf{K}=k \mathbf{I}$ satisfies $k>31$, the system is asymptotically stable at the origin. When selecting the $k=32$, the numerical results, illustrated in Fig. 2, show that the fractional-order unified system (4.2) is driven to its equilibrium point $(0,0,0)$ asymptotically as $t \rightarrow \infty$.
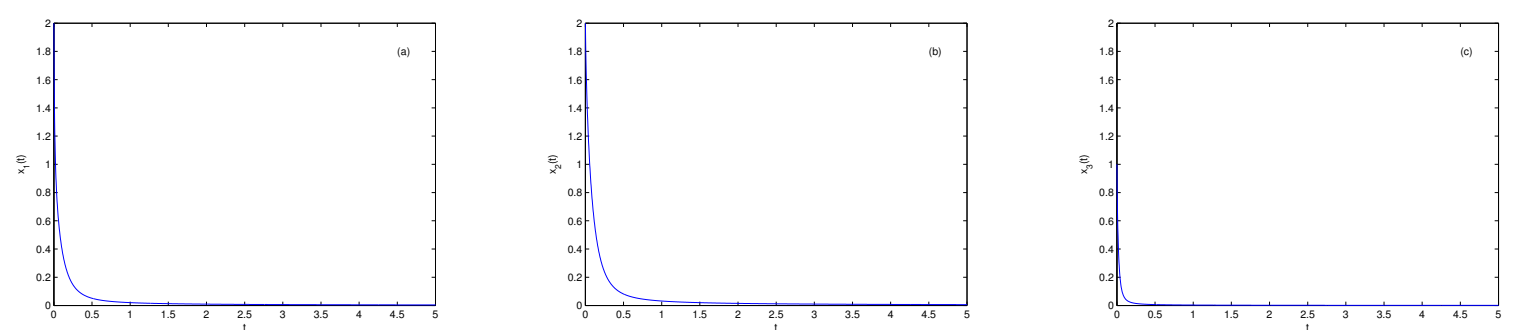

Figure 2: Time waveforms of state variables $x_{1}(a), x_{2}(b)$, and $x_{3}(c)$ of the controlled fractional-order unified system.

\subsection{Stabilization of the fractional-order Liu's system}

In 2009, Liu et al. introduced a 3D chaotic dynamical system which is called Liu's system [25]. Its fractional-order version was described by Bhalekar and Gejji [11], and it has the form: 


$$
{ }_{0} D_{t}^{\alpha} x_{1}(t)=-a x_{1}-e x_{2}^{2}, \quad{ }_{0} D_{t}^{\alpha} x_{2}(t)=b x_{2}-k x_{1} x_{3}, \quad{ }_{0} D_{t}^{\alpha} x_{3}(t)=-c x_{3}+m x_{1} x_{2},
$$

where $a, b, c, e, k, m \in R$. When $a=e=1, b=2.5, k=m=4, c=5$, the system has five equilibrium points. Two of them are complex and three are real equilibriums $(0,0,0),(-0.884,0.940,-0.665)$, and $(-0.884,-0.940,-0.665)$. With the order $\alpha=0.94$ and initial value $(1,1,1)$, the system (4.3) can generate chaos shown in Fig. 3.
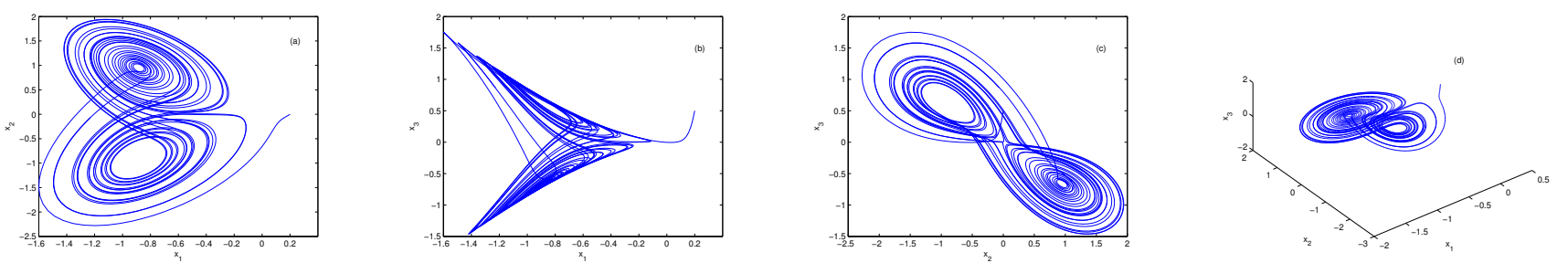

Figure 3: Chaotic attractors in the fractional-order Liu's system with $\alpha=0.94$. The panels (a), (b), (c), and (d) show the $x_{2}-x_{1}$, $x_{3}-x_{1}, x_{3}-x_{2}$, and 3D views, respectively.

The controlled fractional-order chaotic system (4.3) can be rewritten as form (3.4),

$$
{ }_{0} D_{t}^{\alpha} x(t)=A x(t)+g(x(t)) x(t)-K x(t),
$$

where

$$
\mathbf{A}=\left(\begin{array}{ccc}
-\mathrm{a} & 0 & 0 \\
0 & \mathrm{~b} & 0 \\
0 & 0 & -\mathrm{c}
\end{array}\right), \quad \mathbf{g}(\boldsymbol{x}(\mathrm{t}))=\left(\begin{array}{ccc}
0 & -\mathrm{ex} \mathrm{x}_{2} & 0 \\
0 & 0 & -\mathrm{k} x_{1} \\
0 & \mathrm{~m} \mathrm{x}_{1} & 0
\end{array}\right)
$$

Since the system is chaotic, the state variables are bounded, then we can easily obtain $\left|x_{1}(t)\right|<2$ by numerical simulation. It follows $x^{\top}(t) g(x(t)) x(t)=-e x_{1} x_{2}^{2}<2 x_{2}^{2}$ that $c=2$. According to Corollary 3.4, if the matrix $\mathbf{S}=\frac{\mathbf{A}^{\top}+\mathbf{A}}{2}-\mathrm{kI}$ is negative positive, the system (4.2) is asymptotically stable. And we can easily obtain that the maximal eigenvalue of matrix $\frac{A^{\top}+\mathbf{A}}{2}$ is $\lambda_{\max }=2.5$. Hence, if the feedback gain matrix $\mathbf{K}=k \mathbf{I}$ satisfies $k>4.5$, the system is asymptotically stable at the origin. When selecting the $k=5$, the numerical results, illustrated in Fig. 4, show that the fractional-order Liu's system (4.4) is driven to its equilibrium point $(0,0,0)$ asymptotically as $t \rightarrow \infty$.
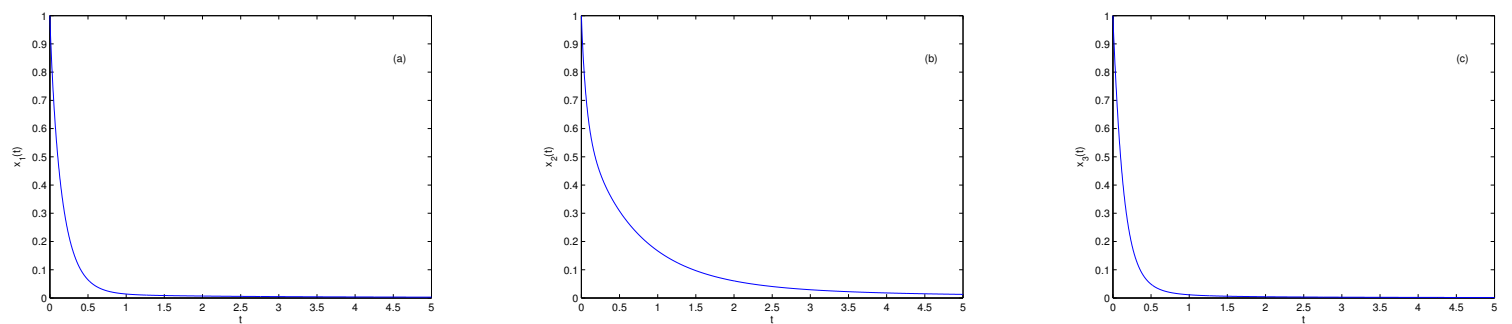

Figure 4: Time waveforms of state variables $x_{1}(a), x_{2}(b)$, and $x_{3}(c)$ of the controlled fractional-order Liu's system.

\subsection{Stabilization of the fractional-order hyperchaotic Chen system}

In 2005, Li et al. [20] proposed a new hyperchaotic Chen system form, which is described by

$$
\dot{x}_{1}=\mathrm{a}\left(\mathrm{x}_{2}-\mathrm{x}_{1}\right)+\mathrm{x}_{4}, \quad \dot{\mathrm{x}}_{2}=\mathrm{d} \mathrm{x}_{1}-\mathrm{x}_{1} \mathrm{x}_{3}+\mathrm{c} \mathrm{x}_{2}, \quad \dot{\mathrm{x}}_{3}=\mathrm{x}_{1} \mathrm{x}_{2}-\mathrm{b} \mathrm{x}_{3}, \quad \dot{\mathrm{x}}_{4}=\mathrm{x}_{2} \mathrm{x}_{3}+\mathrm{r} \mathrm{x}_{4},
$$

where $a, b, c, d, k \in R$. When $a=35, b=3, c=12, d=7$, and $0.085<r \leqslant 0.798$, the system (4.5) is hyperchaotic [42]. Then we consider the fractional version of this system which is given by

$$
{ }_{0} D_{t}^{\alpha} x_{1}=a\left(x_{2}-x_{1}\right)+x_{4}, \quad{ }_{0} D_{t}^{\alpha} x_{2}=d x_{1}-x_{1} x_{3}+c x_{2}, \quad{ }_{0} D_{t}^{\alpha} x_{3}=x_{1} x_{2}-b x_{3}, \quad{ }_{0} D_{t}^{\alpha} x_{4}=x_{2} x_{3}+r x_{4},
$$


where $\alpha$ is the fractional order. When $a=35, b=3, c=12, d=7$, and $r=0.5$, this system has only one equilibrium point at $(0,0,0,0)$. If we consider the commensurate system, according to the numerical simulations, the lowest order of this system to generate hyperchaos is 0.946 . If we select the order $\alpha=0.96$ and initial value $(2,2,1,-1)$, the system exhibits hyperchaotic behavior, as shown in Fig. 5.
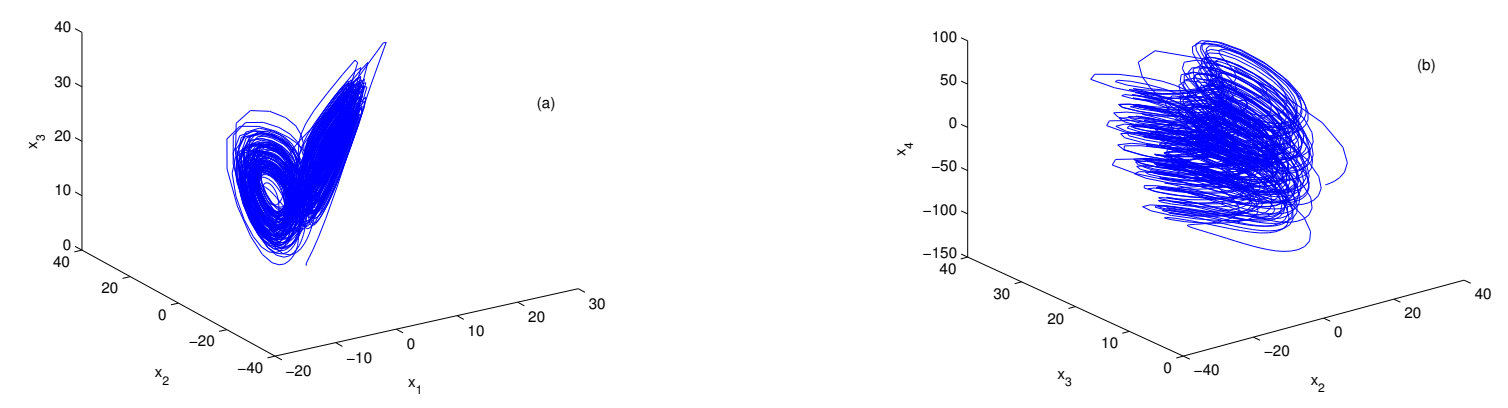

Figure 5: Chaotic attractors in the fractional-order hyperchaotic Chen system with $\alpha=0.96$. The panels (a) and (b) show the 3D views of $x_{3}-x_{2}-x_{1}$ and $x_{4}-x_{3}-x_{2}$, respectively.

The controlled fractional-order chaotic system (4.6) can be rewritten as (3.4),

$$
{ }_{0} D_{t}^{\alpha} x(t)=A x(t)+g(x(t)) x(t)-K x(t),
$$

where

$$
\mathbf{A}=\left(\begin{array}{cccc}
-\mathrm{a} & \mathrm{a} & 0 & 1 \\
\mathrm{~d} & \mathrm{c} & 0 & 0 \\
0 & 0 & -\mathrm{b} & \\
0 & 0 & 0 & \mathrm{r}
\end{array}\right), \quad \mathbf{g}(\boldsymbol{x}(\mathrm{t}))=\left(\begin{array}{cccc}
0 & 0 & 0 & 0 \\
0 & 0 & -\mathrm{x}_{1} & 0 \\
0 & \mathrm{x}_{1} & 0 & 0 \\
0 & 0 & x_{2} & 0
\end{array}\right)
$$

Since the system is chaotic, the state variables are bounded, then we can easily obtain

$$
x^{\top}(t) g(x(t)) x(t)=x_{2} x_{3} x_{4}=\frac{x_{2} x_{3}}{x_{4}} x_{4}^{2} \leqslant 43 x_{4}^{2}
$$

by numerical simulation, i.e., $\mathrm{c}=43$. Hence, according to Corollary 3.4 , if the matrix $\mathbf{S}=\frac{\mathbf{A}^{\top}+\mathbf{A}}{2}-\mathrm{kI}$ is negative positive, the system (4.7) is asymptotically stable. And we can easily obtain that the maximal eigenvalue of matrix $\frac{A^{\top}+\mathbf{A}}{2}$ is $\lambda_{\max } \approx 20$. So if the feedback gain matrix $\mathbf{K}=k \mathbf{I}$ satisfies $k>63$, the system is asymptotically stable at the origin. When selecting the $k=64$, the numerical results, illustrated in Fig. 6 , show that the fractional-order unified system is driven to its equilibrium point $(0,0,0)$ asymptotically as $t \rightarrow \infty$.
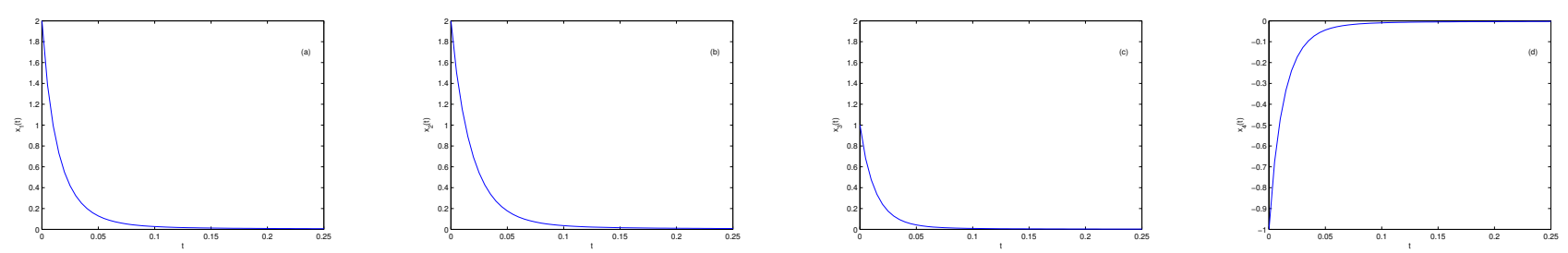

Figure 6: Time waveforms of state variables $x_{1}(a), x_{2}(b) x_{3}(c)$, and $x_{4}(d)$ of the controlled fractional hyperchaotic Chen system.

\section{Conclusion}

In this letter we proposed a fractional-order controller and presented a control law for the arbitrary dimensional fractional-order systems. According to the Lyapunov stability theorem of the fractionalorder systems, the control law achieved to stabilize the fractional-order chaotic and hyperchaotic systems. 
The proposed method provides a theoretical basis for the applications of the fractional-order control in fractional dynamic systems. Our proposed criteria are very simple, universal, and could be easily applied. Furthermore, this method can control fractional-order chaotic and hyperchaotic systems more effectively and quickly than traditional controllers. We have implemented and verified our method for other fractional-order chaotic and hyperchaotic systems [23, 29, 30, 32] namely: the fractional-order NewtonLeipnik's system, the Lü's chaotic system, financial system, hyperchaotic Rössler system, and etc.. The numerical simulation results indicate that the proposed controller can effectively control the fractionalorder chaotic and hyperchaotic systems stable, and the proposed method provides a theoretical basis for the applications of the fractional-order control in fractional-order dynamic systems.

In the future works about this topic, we will consider whether the proposed method can be extended to the control of other complex chaotic systems, such as fractional multi-scroll chaotic systems [27], and the networked fractional chaotic systems [8, 35, 40,45]. The multi-scroll chaotic systems have non-smooth functions and complex multi-scroll chaotic attractors which make systems much more complex than the similar fractional chaotic systems. The networked fractional chaotic systems are composed of many nodes, and each node receives information from its adjacent nodes. Extending the proposed method to these system is important for both control theory and application of fractional chaotic system.

\section{Acknowledgment}

Our deepest gratitude goes to the anonymous reviewers for their careful work and thoughtful suggestions that have helped to improve this paper substantially. This work is partly supported by Natural Science Foundation of China (Grant No.11701397, 61573010), Sichuan Youth Science and Technology Foundation (Grant No.2016JQ0046), Artificial Intelligence Key Laboratory of Sichuan Province (Grant No.2016RYJ06), Found of Sichuan University of Science and Engineering (Grant No.2016RCL33, 2015RC10), Opening Project of Key Laboratory of Higher Education of Sichuan Province for Enterprise Informationalization and Internet of Things (Grant No.2016WYJ04).

\section{References}

[1] W. M. Ahmad, R. El-Khazali, Y. Al-Assaf, Stabilization of generalized fractional order chaotic systems using state feedback control, Chaos, Solitons \& Fractals, 22 (2004), 141-150. 1

[2] Z.-B. Bai, On solutions of some fractional m-point boundary value problems at resonance, Electron. J. Qual. Theory Differ. Equ., 2010 (2010), 15 pages. 1

[3] Z.-B. Bai, Solvability for a class of fractional m-point boundary value problem at resonance, Comput. Math. Appl., 62 (2011), 1292-1302. 1

[4] Z.-B. Bai, X.-Y. Dong, C. Yin, Existence results for impulsive nonlinear fractional differential equation with mixed boundary conditions, Bound. Value Probl., 2016 (2016), 11 pages. 1

[5] Z.-B. Bai, T.-T. Qiu, Existence of positive solution for singular fractional differential equation, Appl. Math. Comput., 215 (2009), 2761-2767. 1

[6] Z.-B. Bai, Y.-H. Zhang, The existence of solutions for a fractional multi-point boundary value problem, Comput. Math. Appl., 60 (2010), 2364-2372. 1

[7] T. A. Burton, Fractional differential equations and Lyapunov functionals, Nonlinear Anal., 74 (2011), 5648-5662. 1

[8] Y. Chen, J.-H. Lü, Z.-L. Lin, Consensus of discrete-time multi-agent systems with transmission nonlinearity, Automatica J., 49 (2013), 1768-1775. 5

[9] K. Diethelm, The analysis of fractional differential equations, Springer, Berlin, (2004). 1

[10] Z. M. Ge, W. R. Jhuang, Chaos, control and synchronization of a fractional order rotational mechanical system with a centrifugal governor, Chaos, Solitons \& Fractals, 33 (2007), 270-289. 1

[11] S. Bhalekar, V. Daftardar-Gejji, Fractional ordered Liu system with time-delay, Commun. Nonlinear Sci. Numer. Simul., 15 (2010), 2178-2191. 4.2

[12] R. Gorenflo, F. Mainardi, D. Moretti, P. Paradisi, Time fractional diffusion: A discrete random walk approach, Nonlinear Dynamic, 29 (2002), 129-143. 2.1

[13] I. Grigorenko, E. Grigorenko, Chaotic dynamics of the fractional Lorenz system, Physical Review Letters, 2003 (2003), 4 pages. 1

[14] L.-L. Huang, S.-J. He, Stability of fractional state space system and its application to fractional order chaotic system, Acta Physica Sinica, 60 (2011), 447031-447036. 1 
[15] V. Lakshmikantham, S. Leela, M. Sambandham, Lyapunov theory for fractional differential equations, Commun. Appl. Anal., 12 (2008), 365-376. 1

[16] V. Lakshmikantham, S. Leela, S. J. Vasundara Devi, Theory of fractional dynamic systems, Cambridge Scientific Publishers, Cambridge, (2009). 1

[17] C.-G. Li, G.-R. Chen, Chaos in the fractional order Chen system and its control, Chaos, Solitons \& Fractals, 22 (2004), 549-554. 1

[18] Y. Li, Y.-Q. Chen, I. Podlubny, Stability of fractional-order nonlinear dynamic systems: Lyapunov direct method and generalized Mittag Leffler stability, Comput. Math. Appl., 59 (2010), 1810-1821. 1, 2.3

[19] X.-P. Li, X.-Y. Lin, Y.-Q. Lin, Lyapunov-Type Conditions and Stochastic Differential Equations Driven By G-Brownian Motion, J. Math. Anal. Appl., 439 (2016), 235-255. 1

[20] Y.-X. Li, W. K. Tang, G.-R. Chen, Generating hyperchaos via state feedback control, International Journal of Bifurcation and Chaos, 15 (2005), 3367-3371. 1, 4.3

[21] T.-Z. Li, Y. Wang, M.-K. Luo, Control of chaotic and hyperchaotic systems based on a fractional order controller, Chinese Physics B, 2014 (2014), 12 pages. 4.1

[22] F. Li, C.-N. Wang, J. Ma, Reliability of linear coupling synchronization of hyperchaotic systems with unknown parameters, Chinese Phys. B, 2013 (2013), 9 pages. 1

[23] T.-Z. Li, Y. Wang, Y. Yong, Designing synchronization schemes for fractional-order chaoticsystem via a single state fractional-order controller, Optik, 125 (2014), 6700-6705. 5

[24] C.-X. Liu, L. Liu, A novel four-dimensional autonomous hyperchaotic system, Chinese Phys. B, 18 (2009), 2188-2193. 1

[25] C.-X. Liu, L. Liu, T. Liu, A novel three-dimensional autonomous chaos system, Chaos, Solitons \& Fractals, 39 (2009), 1950-1958. 4.2

[26] J.-H. Lü, G.-R. Chen, A new chaotic attractor coined, Internat. J. Bifur. Chaos Appl. Sci. Engrg., 12 (2002), 659-661. 4.1

[27] J.-H. Lü, G.-R. Chen, Generating multiscroll chaotic attractors: theories, methods and applications, Internat. J. Bifur. Chaos Appl. Sci. Engrg., 16 (2006), 775-858. 5

[28] D. Matignon, Representations en variables detat de modeles de guides dondes avec drivation fractionnaire [Ph.D. thesis], Universit Paris, (1994). 1

[29] A. Oustaloup, J. Sabatier, P. Lanusse, From fractal robustness to the CRONE control, Fract. Calc. Appl. Anal., 2 (1999), 1-30. 5

[30] I. Petras, Fractional-order Nonlinear Systems: Modeling, Analysis and Simulation, Higher Education Press, Beijing, (2011). 1, 2.1, 2.2, 2.7, 5

[31] I. Podlubny, Fractional Differential Equations, Academic Press, New York, (1999). 1, 2.1, 2.2, 2.6, 2.7

[32] O. E. Rössler, An equation for hyperchaos, Phys. Lett. A, 71 (1979), 155-157. 1, 5

[33] J. J. Slotine, W. Li, Applied nonlinear control, Prentice Hall, London, (1991). 3

[34] Z. Wang, A Numerical Method For Delayed Fractional-Order Differential Equations, J. Appl. Math., 2013 (2013), 17 pages. 2.1

[35] S.-D. Wang, Y. Chen, Q.-Y. Wang, E. Li, Y.-S. Su, D. Meng, Analysis For Gene Networks Based On Logic Relationships, J. Syst. Sci. Complex., 23 (2010), 999-1011. 5

[36] Z. Wang, X. Huang, Y.-X. Li, X.-N. Song, A new image encryption algorithm based on fractional-order hyperchaotic Lorenz system, Chinese Phys. B, 2013 (2013), 8 pages. 1

[37] Z. Wang, X. Huang, G.-D. Shi, Analysis of Nonlinear Dynamics and Chaos In A Fractional Order Financial System With Time Delay, Comput. Math. Appl., 62 (2011), 1531-1539. 1

[38] Z. Wang, X. Huang, J.-P. Zhou, A Numerical Method For Delayed Fractional-Order Differential Equations: Based On G-L Definition, Appl. Math. Inf. Sci., 7 (2013), 525-529. 2.1

[39] Y. Wang, T.-Z. Li, Stability analysis of fractional-order nonlinear systems with delay, Math. Probl. Eng., 2014 (2014), 8 pages. 1

[40] Y. Wang, T.-Z. Li, Synchronization of fractional order complex dynamical networks, Phys. A, 428 (2015), 1-12. 5

[41] X.-J. Wang, J. Li, G.-R. Chen, Chaos in the fractional order unified system and its synchronization, J. Franklin Inst., 345 (2008), 392-401. 4.1

[42] X.-Y. Wang, M.-J. Wang, A hyperchaos generated from Lorenz system, Phys. A, 387 (2008), 3751-3758. 4.3

[43] N.-N. Yang, C.-X. Liu, C.-J. Wu, A hyperchaotic system stabilization via inverse optimal control and experimental research, Chinese Physics B, 2010 (2010), 10 pages. 1

[44] S. Yuste, J. Murillo, On three explicit difference schemes for fractional diffusion and diffusionwave equations, Physicas Scripta, 2009 (2009), 7 pages. 2.1

[45] Y.-L. Zhang, K.-B. Lv, S.-D. Wang, J.-L. Su, D.-Z. Meng, Modeling Gene Networks In Saccharomyces Cerevisiae Based On Gene Expression Profiles, Comput. Math. Methods Med., 2015 (2015), 10 pages. 5

[46] R.-X. Zhang, S.-P. Yang, Chaos in the fractional-order conjugate Chen system and its circuit emulation, Acta Physica Sinica, 58 (2009), 29571-29576. 1

[47] Q.-S. Zhong, Impulsive Control for Fractional-Order Chaotic Systems, Chinese Phys. Lett., 25 (2008), 2812-2815. 1 